\title{
Ação nematicida do eugenol em tomateiro ${ }^{1}$
}

\author{
Lara Caroline Borges Moreira ${ }^{2}$, Bruno Sérgio Vieira ${ }^{3}$, \\ Célio Vinícius da Mota Júnior ${ }^{2}$, Everaldo Antônio Lopes ${ }^{4}$, Ellen Júnia Canedo²
}

\begin{abstract}
Nematicidal effect of eugenol on tomato plants

Essential oils from several plant species have nematicidal properties and could be used for controlling root-knot nematodes. Thus, this study aimed at evaluating the effect of eugenol concentrations on the control of Meloidogyne incognita and Meloidogyne javanica, in a greenhouse. The eugenol was applied at the concentrations of $0.25 \mathrm{~mL} \mathrm{~L}^{-1}, 0.5 \mathrm{~mL} \mathrm{~L}^{-1}, 0.75 \mathrm{~mL} \mathrm{~L}^{-1}$ and $1.0 \mathrm{~mL} \mathrm{~L}^{-1}$, as a foliar spray or soil drench. A complete randomized design, in a $4 \times 2+1$ factorial scheme (concentrations of eugenol $\mathrm{x}$ methods of application + control - additional treatment), with five replications, was used. The number of galls and eggs of $M$. incognita and eggs of $M$. javanica decreased in plants treated with eugenol, when compared to the control. Soil drench was the most efficient method for applying eugenol, regarding the reduction in the number of galls. Increasing concentrations of eugenol reduced the number of $M$. incognita eggs. Eugenol showed potential to be used in the management of root-knot nematodes, though further studies are necessary to support such an expectation.
\end{abstract}

KEY-WORDS: Solanum lycopersicum L.; Meloidogyne incognita Chitwood and M. javanica Treub; Syzygium aromaticum (L.) Merr. and Perry; root-knot nematode.

\section{INTRODUÇÃO}

Acultura do tomateiro (Solanum lycopersicum L.) pode ser atacada por inúmeras pragas e patógenos, principalmente em países tropicais, como o Brasil. Dentre os organismos que parasitam o tomateiro, os nematoides das galhas, principalmente as espécies Meloidogyne incognita (Kofoid \& White) Chitwood e Meloidogyne javanica (Treub) Chitwood, estão entre os mais danosos (Charchar \& Aragão 2005). Em todo o mundo, perdas causadas por estes patógenos va-

\section{RESUMO}

Óleos essenciais de várias espécies de plantas possuem propriedades nematicidas e poderiam ser utilizados no controle do nematoide das galhas. Desta forma, esta pesquisa objetivou avaliar o efeito da aplicação de concentrações de eugenol no controle de Meloidogyne incognita e Meloidogyne javanica, em casa-de-vegetação. $\mathrm{O}$ eugenol foi aplicado nas concentrações de $0,25 \mathrm{~mL} \mathrm{~L}^{-1}, 0,50 \mathrm{~mL} \mathrm{~L}^{-1}, 0,75 \mathrm{~mL} \mathrm{~L}^{-1}$ e $1,00 \mathrm{~mL} \mathrm{~L}^{-1}$, na forma de pulverização foliar ou adição ao solo. O delineamento experimental foi inteiramente casualizado, em arranjo fatorial $4 \times 2+1$ (concentrações de eugenol x formas de aplicação + testemunha - tratamento adicional), com cinco repetições. Os números de galhas e de ovos de $M$. incognita e de ovos de M. javanica foram reduzidos em plantas tratadas com eugenol, em comparação com a testemunha. A adição ao solo foi o método mais eficiente de aplicação do eugenol, considerando-se a redução no número de galhas. Houve efeito significativo do aumento de concentrações de eugenol na redução do número de ovos de $M$. incognita. O eugenol apresenta potencial para ser utilizado no manejo do nematoide das galhas, embora novos estudos ainda sejam necessários para suportar tal expectativa.

PALAVRAS-CHAVE: Solanum lycopersicum L.; Meloidogyne incognita Chitwood e M. javanica Treub; Syzygium aromaticum (L.) Merr. e Perry; fitonematoides.

riam de acordo com o nível de infestação do solo e de susceptibilidade do hospedeiro, condições edafoclimáticas e manejo adotado pelos produtores (Moens et al. 2009).

Os métodos tradicionalmente recomendados para o controle de fitonematoides são a rotação de culturas e o uso de nematicidas e de variedades resistentes (Ferraz et al. 2010). A rotação de culturas é opção importante no manejo do patógeno, no entanto, a polifagia do nematoide das galhas é um dos fatores que limitam a escolha de culturas não hospedeiras, para inclusão no esquema de rotação.

1. Trabalho recebido em jan./2013 e aceito para publicação em ago./2013 (nº registro: PAT 22293).

2. Centro Universitário de Patos de Minas (Unipam), Patos de Minas, MG, Brasil.E-mails: laracaroline2009@hotmail.com, cvmjagro@gmail.com, ellencanedo@hotmail.com.

3. Universidade Federal de Uberlândia (UFU), Monte Carmelo, MG, Brasil.E-mail: brunovieira@iciag.ufu.br.

4. Universidade Federal de Viçosa (UFV), Rio Paranaíba, MG, Brasil. E-mail: everaldolopes@ufv.br. 
Os nematicidas são prejudiciais à saúde humana e ao meio ambiente, além de apresentarem custo elevado (Nico et al. 2004). O plantio de genótipos resistentes a nematoides é altamente recomendado para qualquer cultivo agrícola, desde que tais materiais estejam disponíveis para os produtores e que apresentem características agronômicas desejáveis. Todavia, tal panorama não se aplica aos genótipos de tomateiro para mesa (Ferraz et al. 2010). Assim, novas estratégias de manejo de fitonematoides devem ser continuamente pesquisadas, a exemplo do uso de óleos essenciais de plantas (Ferraz \& Freitas 2004).

Substâncias tóxicas aos nematoides podem ser produzidas durante o metabolismo secundário de diversas espécies de plantas, constituindo-se em matéria-prima potencial para a produção de nematicidas naturais (Chitwood 2002, Ferraz \& Freitas 2004, Lopes et al. 2005). Um exemplo de substância com potencial nematicida é o eugenol (Bala \& Sukul 1987), óleo essencial extraído do cravo-da-índia (Syzygium aromaticum (L.) Merr. \& Perry) e utilizado rotineiramente em procedimentos odontológicos.

O presente estudo objetivou avaliar o efeito da forma de aplicação e concentrações de eugenol no controle de $M$. incognita e M. javanica, em casa-de-vegetação.

\section{MATERIAL E MÉTODOS}

Os experimentos foram conduzidos em casa-de-vegetação do Centro Universitário de Patos de Minas (Unipam), em Patos de Minas (MG), de novembro de 2011 a fevereiro de 2012.

Plântulas de tomateiro 'Santa Clara' de 21 dias de idade foram transplantadas para vasos plásticos de 2,0 L de capacidade, preenchidos com mistura de terriço e areia, na proporção de 1:1 (v:v). O substrato para cultivo das plantas foi autoclavado por duas vezes, a $120^{\circ} \mathrm{C}$, por uma hora.

Aos dez dias após o transplantio, o solo foi infestado com 5.000 ovos e eventuais juvenis de M. incognita ou M. javanica, por meio da adição de 5,0 $\mathrm{mL}$ da suspensão de inóculo a três orifícios feitos ao redor da planta. Os ovos dos nematoides foram extraídos pela técnica de Hussey \& Barker (1973), modificada por Boneti \& Ferraz (1981), a partir de raízes de tomateiros previamente infectadas com populações puras dos nematoides, mantidas em casa-de-vegetação.
Decorridos dez dias da infestação do solo com os nematoides, o eugenol foi aplicado, por meio de pulverização, à parte aérea das plantas, ou por adição direta à superfície do solo, na base das plantas. Em ambas as formas de aplicação, as concentrações do óleo essencial foram de $0,25 \mathrm{~mL} \mathrm{~L}^{-1}, 0,50 \mathrm{~mL} \mathrm{~L}^{-1}$, $0,75 \mathrm{~mL} \mathrm{~L}^{-1}$ e $1,00 \mathrm{~mL} \mathrm{~L}^{-1}$.

O eugenol utilizado foi extraído de cravo-da-índia adquirido em estabelecimento comercial especializado em produtos odontológicos. As pulverizações foram realizadas conforme descrito por Lopes et al. (2005), com o envolvimento dos vasos em sacos plásticos, evitando-se que o extrato pulverizado na parte aérea da planta, por meio de pulverizador manual, atingisse o solo. As aplicações foram repetidas após dez dias. Plantas inoculadas com os nematoides e que não receberam nenhuma forma de tratamento com eugenol foram mantidas como testemunhas.

$\mathrm{O}$ delineamento experimental adotado foi do tipo inteiramente casualizado (DIC), em arranjo fatorial 4 × $2+1$ (concentrações de eugenol x formas de aplicação + testemunha - tratamento adicional), com cinco repetições. Cada espécie de nematoide foi avaliada em experimento individualizado. A parcela experimental foi constituída de um vaso contendo uma planta de tomateiro.

Realizou-se o manejo de pragas e doenças pela aplicação do inseticida espinosade $\left(15{\mathrm{~mL} 100 \mathrm{~L}^{-1} \mathrm{de}}\right.$ água) e a mistura clorotalonil + oxicloreto de cobre (350 g $\left.100 \mathrm{~L}^{-1}\right)$. As plantas daninhas foram retiradas manualmente. O turno de rega adotado foi o diário, optando-se por uso de regador e mantendo-se o solo com, aproximadamente, $60 \%$ da capacidade de campo.

As avaliações dos experimentos ocorreram aos 60 dias após a infestação do solo com os nematoides, determinando-se o número de galhas e de ovos dos patógenos por sistema radicular.

As análises estatísticas foram realizadas com o auxílio do programa estatístico Assistat 7.6 (Silva 2011). Os dados obtidos foram submetidos aos testes de normalidade (Teste de Shapiro-Wilks) e de homogeneidade do erro experimental (Teste de Levene), transformados para $\log _{10}(\mathrm{X})$ e submetidos à análise de variância. A comparação entre a testemunha e os demais tratamentos foi realizada pelo teste de Dunnett. Na ausência de interação entre os fatores, os efeitos das formas de aplicação do eugenol e das diferentes concentrações do óleo essencial foram 
analisados por meio do teste $\mathrm{F}$ e análise de regressão, respectivamente. O nível de significância de 5\% foi adotado em todos os procedimentos estatísticos.

\section{RESULTADOS E DISCUSSÃO}

O número de galhas e de ovos de $M$. incognita foi significativamente reduzido em raízes de tomateiro tratadas com eugenol, em comparação com a testemunha não tratada, independentemente do modo de aplicação e da dose de óleo essencial (Tabela 1). A redução no número de galhas do patógeno foi $67,60 \%$ maior, quando o eugenol foi aplicado diretamente ao solo, em detrimento à pulverização foliar, independentemente da dose do produto. Por outro lado, não houve diferença significativa na redução do número de ovos, em função do modo de aplicação, mas sim em resposta a concentrações crescentes do eugenol, conforme o modelo $\mathrm{Y}=7.421,1875-4.681,75 . \mathrm{X}$ (Tabela 1).

A aplicação de eugenol não reduziu o número de galhas de $M$. javanica, quando comparado com a testemunha, independentemente da dose (Tabela 2). Pelo contrário, em parcelas onde o produto foi aplicado ao solo ou às folhas na dose de $1,0 \mathrm{~mL} \mathrm{~L}^{-1} \mathrm{e}$ às folhas na dose de $0,25 \mathrm{~mL} \mathrm{~L}^{-1}$, o número de galhas foi maior do que na testemunha não tratada.

Ao contrário do observado para o número de galhas, todos os tratamentos com eugenol reduziram o número de ovos de $M$. javanica, em comparação com parcelas não tratadas. No entanto, não houve efeito significativo das formas de aplicação, tampouco das concentrações do produto sobre a variável em questão (Tabela 2).

Tabela 1. Número de galhas e ovos de Meloidogyne incognita, em raízes de tomateiro, após a pulverização foliar ou a adição ao solo de diferentes concentrações de eugenol, aos 60 dias após a infestação do solo com 5.000 ovos do nematoide (Patos de Minas, MG, 2012).

\begin{tabular}{|c|c|c|c|c|c|c|}
\hline \multirow{2}{*}{$\begin{array}{l}\text { Concentração de eugenol } \\
\qquad\left(\mathrm{mL} \mathrm{L}^{-1}\right)\end{array}$} & \multicolumn{3}{|c|}{ Número de galhas ${ }^{1}$} & \multicolumn{3}{|c|}{ Número de ovos ${ }^{1}$} \\
\hline & $\begin{array}{l}\text { Pulverização } \\
\text { foliar }\end{array}$ & $\begin{array}{l}\text { Adição ao } \\
\text { solo }\end{array}$ & Média $^{2}$ & $\begin{array}{l}\text { Pulverização } \\
\text { foliar }\end{array}$ & $\begin{array}{l}\text { Adição ao } \\
\text { solo }\end{array}$ & Média $^{3}$ \\
\hline 0,25 & $221 *$ & $84 *$ & 152 & $7.099^{*}$ & $5.750 *$ & 6.424 \\
\hline 0,50 & $158^{*}$ & $64 *$ & 111 & $4.849^{*}$ & $5.525^{*}$ & 5.187 \\
\hline 0,75 & $111^{*}$ & $48^{*}$ & 80 & $3.600^{*}$ & $2.750 *$ & 3.175 \\
\hline 1,00 & $229 *$ & $40^{*}$ & 134 & $4.025^{*}$ & $2.362 *$ & 3.194 \\
\hline Média $^{4}$ & $180 \mathrm{a}$ & $59 \mathrm{~b}$ & - & $4.893^{\text {ns }}$ & 4.097 & - \\
\hline Testemunha & \multicolumn{2}{|c|}{303} & \multicolumn{4}{|c|}{23.999} \\
\hline CV (\%) & \multicolumn{2}{|c|}{7,53} & \multicolumn{4}{|c|}{34,40} \\
\hline
\end{tabular}

Tabela 2. Número de galhas e ovos de Meloidogyne javanica, em raízes de tomateiro, após a pulverização foliar ou adição ao solo de diferentes concentrações de eugenol, aos 60 dias após a infestação do solo com 5.000 ovos do nematoide (Patos de Minas, MG, 2012).

\begin{tabular}{|c|c|c|c|c|c|c|}
\hline \multirow{2}{*}{$\begin{array}{l}\text { Concentração de eugenol } \\
\qquad\left(\mathrm{mL} \mathrm{L}^{-1}\right)\end{array}$} & \multicolumn{3}{|c|}{ Número de galhas ${ }^{1}$} & \multicolumn{3}{|c|}{ Número de ovos ${ }^{1}$} \\
\hline & $\begin{array}{c}\text { Pulverização } \\
\text { Foliar }\end{array}$ & $\begin{array}{c}\text { Adição ao } \\
\text { solo }\end{array}$ & Média $^{2}$ & $\begin{array}{c}\text { Pulverização } \\
\text { Foliar }\end{array}$ & $\begin{array}{c}\text { Adição ao } \\
\text { solo }\end{array}$ & Média $^{2}$ \\
\hline 0,25 & $221 *$ & 63 & 122 & $11.993^{*}$ & $2.406^{*}$ & 7.199 \\
\hline 0,50 & 158 & 64 & 137 & $7.000 *$ & $5.650 *$ & 6.325 \\
\hline 0,75 & 111 & 106 & 143 & $7.967 *$ & $6.110^{*}$ & 7.038 \\
\hline 1,00 & $229 *$ & $186^{*}$ & 187 & $10.900 *$ & $5.630 *$ & 8.265 \\
\hline Média $^{3}$ & $190 \mathrm{a}$ & $105 \mathrm{~b}$ & - & $9.465^{\mathrm{ns}}$ & 4.949 & - \\
\hline Testemunha & \multicolumn{2}{|c|}{90,2} & \multicolumn{4}{|c|}{61.766} \\
\hline CV $(\%)$ & \multicolumn{2}{|c|}{17,32} & \multicolumn{4}{|c|}{13,40} \\
\hline
\end{tabular}


O eugenol é um composto fenólico volátil (Kamatou et al. 2012), que possui atividade inseticida (Liska et al. 2010), bactericida (Oyedemi et al. 2009), fungicida (Carrasco et al. 2012) e alelopática (Mazzafera 2003). Além disto, os resultados do presente trabalho suportam a hipótese de que a substância é capaz de matar ou inibir o desenvolvimento de fitonematoides, em plantas hospedeiras (Tsao \& Yu 2000, Park et al. 2007).

Os mecanismos de ação do eugenol, no controle de M. incognita e M. javanica, nesta pesquisa, estão possivelmente relacionados com a ação direta da substância, causando a morte do patógeno por contato e/ou de maneira indireta, por meio da ativação da resistência sistêmica da planta (Chitwood 2002).

A aplicação de eugenol ao solo pode ter afetado, de forma direta, a embriogênese, ou causado a morte dos juvenis de segundo estádio dos nematoides, visto que as propriedades anti-helmínticas do composto já foram comprovadas (Tsao \& Yu 2000, Park et al. 2007). No entanto, a atividade nematicida da substância pode variar em função da espécie de nematoide avaliada, uma vez que a redução no número de galhas foi observada em parcelas infestadas com M. incognita (Tabela 1), ao contrário do ocorrido com $M$. javanica (Tabela 2).

Considerando-se que o número de galhas está diretamente relacionado com a quantidade de nematoides que penetraram e estabeleceram sítios de alimentação no hospedeiro, possivelmente M. incognita seja mais sensível à ação do eugenol do que $M$. javanica. Todavia, a hipótese de sensibilidade diferencial entre espécies de Meloidogyne ao eugenol deve ainda ser avaliada, assim como os mecanismos que governam o fenômeno, caso ocorra.

A aplicação de eugenol na parte aérea das plantas foi realizada com o intuito de avaliar o possível efeito nematicida sistêmico da substância, tal qual sugerido por Bala \& Sukul (1987). Os referidos autores verificaram que a aplicação de eugenol à parte aérea de quiabeiros (Abelmoschus esculentum (L.) Moench.), na dose de $0,5 \mathrm{~mL} \mathrm{~L}^{-1}$, reduziu o número de galhas de $M$. incognita, mesmo que o produto não tivesse entrado em contato com o solo infestado, indicando efeito sistêmico.

A pulverização do óleo essencial, na presente pesquisa, também reduziu o número de galhas e ovos de $M$. incognita e de ovos de $M$. javanica, na cultura do tomateiro (Tabelas 1 e 2), corroborando os resultados de Bala \& Sukul (1987). Em outros estudos, a pulverização de extratos aquosos de folhas de manjericão (Ocimum basilicum L.), planta que contém eugenol, na parte aérea de tomateiros, reduziu em 32,5\% o número de galhas de $M$. incognita (Lopes et al. 2005), mas nenhum efeito significativo foi verificado em plantas infectadas com $M$. javanica (Lopes et al. 2005, Gardiano et al. 2008).

O uso de compostos capazes de serem absorvidos pelas folhas, translocados e liberados pelas raízes poderia ser importante estratégia de manejo de nematoides (Lopes et al. 2005). No entanto, a descoberta de substâncias com tais características é difícil, uma vez que a sistemicidade via simplasto até as raízes é rara, entre as moléculas usadas como defensivos agrícolas, com predominância da translocação via apoplasto (Zambolim 2008). O oxamyl é um exíguo exemplo de nematicida capaz de penetrar pela cutícula das folhas e ser translocado até as raízes, via floema, e atuar no controle de nematoides (Lawrence \& McLean 2002).

Os possíveis efeitos da absorção e translocação do eugenol em plantas ainda devem ser investigados. No entanto, a raridade do fenômeno da translocação basipetal de moléculas aplicadas sobre as plantas sugere que outros mecanismos estejam envolvidos no efeito do eugenol no controle de nematoides, como, por exemplo, a ativação de mecanismos de defesa da planta (Schwan-Estrada et al. 2003, Salgado \& Silva 2005).

A aplicação de substâncias químicas, ou agentes bióticos, pode induzir resistência sistêmica em plantas a diversos patógenos (Cavalcanti et al. 2005), a exemplo dos nematoides (Salgado \& Silva 2005). Birch et al. (1993), por exemplo, reportaram a ação sistêmica do açúcar 2,5-dihidroximetil-3,4-dihidroxipirrolidina (DMDP), isolado de plantas do gênero Lonchocarpus e Derris, no controle de várias espécies de nematoides, e atribuíram o efeito observado à indução de resistência. Em outro estudo, a pulverização com mistura aquosa contendo $0,2 \%$ de óleo de Argemone mexicana L. reduziu o número de galhas e de juvenis de M. incognita no solo e aumentou o crescimento de quiabeiros, com ação mais pronunciada da aplicação foliar do que da adição ao solo (Das \& Sukul 1988). Para a comprovação do papel do eugenol na ativação de mecanismos de resistência de tomateiros ao nematoide das galhas, portanto, faz-se necessário avaliar possíveis alterações bioquímicas, celulares e estruturais da planta tratada com o óleo essencial e atacada pelo patógeno (Salgado \& Silva 2005). 
Assim, novos estudos devem ser conduzidos a campo, para confirmar o potencial do eugenol no manejo do nematoide das galhas, assim como deve ser considerada a plena compreensão dos mecanismos de ação envolvidos.

\section{CONCLUSÕES}

1. A aplicação de eugenol reduziu o número de galhas e ovos de M. incognita e de ovos de M. javanica, em comparação com a testemunha.

2. A adição ao solo foi o método mais eficiente de aplicação do eugenol, principalmente considerando-se a redução no número de galhas de ambas as espécies de nematoides.

\section{REFERÊNCIAS}

BALA, S. K.; SUKUL, N. C. Systemic nematicidal effect of eugenol. Nematropica, DeLeon Springs, v. 17, n. 2, p. 219-222, 1987.

BIRCH, A. N. E. et al. DMDP: a plant-derived sugar analogue with systemic activity against plant parasitic nematodes. Nematologica, Leiden, v. 39, n. 1-4, p. 521535, 1993.

BONETI, J. I. S.; FERRAZ, S. Modificação do método de Hussey e Barker para a extração de ovos de Meloidogyne exigua de cafeeiro. Fitopatologia Brasileira, Brasília, DF, v. 6, n. 3, p. 553, 1981. Suplemento.

CARRASCO, H. et al. Antifungal activity of eugenol analogues: influence of different substituents and studies on mechanism of action. Molecules, Basel, v. 17, n. 1, p. 1002-1024, 2012.

CAVALCANTI, L. S.; BRUNELLI, K. R.; STARGARLIN, J. R. Aspectos bioquímicos e moleculares da resistência induzida. In: CAVALCANTI, L. S. et al. (Eds.). Indução de resistência em plantas a patógenos e insetos. Piracicaba: Fealq, 2005.

CHARCHAR, J. M.; ARAGÃO, F. A. S. Reprodução de Meloidogyne spp. em cultivares de tomate e pepino sob estufa plástica e campo. Nematologia Brasileira, Piracicaba, v. 29, n. 2, p. 243-249, 2005.

CHITWOOD, D. J. Phytochemical based strategies for nematode control. Annual Review of Phytopathology, Palo Alto, v. 40, n. 1, p. 221-249, 2002.

DAS, S.; SUKUL, N. C. Nematicidal effect of the oil from the seeds of Argemone mexicana. Environment and Ecology, Kalyani, v. 6, n. 1, p. 194-197, 1988.
FERRAZ, S.; FREITAS, L. G. Use of antagonistic plants and natural products. In: CHEN, Z.; CHEN, S.; DICKSON, D. W. (Eds.). Nematology: advances and perspectives. Beijing: Tsinghua University Press, 2004. p. 931-978.

FERRAZ, S. et al. Manejo sustentável de fitonematoides. Viçosa: Ed. da UFV, 2010.

GARDIANO, C. G. et al. Avaliação de extratos aquosos de espécies vegetais, aplicados via pulverização foliar, sobre Meloidogyne javanica. Summa Phytopathologica, Botucatu, v. 34, n. 4, p. 376-377, 2008.

HUSSEY, R. S.; BARKER, K. R. A. Comparison of methods of collecting inocula of Meloidogyne spp. including a new technique. Plant Disease Reporter, Saint Paul, v. 57, n. 12, p. 1025-1028, 1973.

KAMATOU, G. P.; VERMAAK, I.; VILJOEN, A. M. Eugenol: from the remote Maluku Islands to the international market place: a review of a remarkable and versatile molecule. Molecules, Basel, v. 17, n. 6, p. 69536981, 2012.

LAWRENCE, G. W.; MCLEAN, K. S. Foliar applications of oxamyl with aldicarb for the management of Meloidogyne incognita on cotton. Nematropica, DeLeon Springs, v. 32, n. 2, p. 103-112, 2002.

LISKA, A. et al. Contact and fumigant activity of 1,8-cineole, eugenol and camphor against Tribolium castaneum (Herbst). Julius-Kühn-Archiv, Berlin, v. 425, n. 1, p. 716-720, 2010.

LOPES, E. A. et al. Efeito dos extratos aquosos de mucuna preta e de manjericão sobre Meloidogyne incognita e $M$. javanica. Nematologia Brasileira, Piracicaba, v. 29, n. 1, p. $67-74,2005$.

MAZZAFERA, P. Efeito alelopático do extrato alcoólico do cravo-da-índia e eugenol. Revista Brasileira de Botânica, São Paulo, v. 26, n. 2, p. 231-238, 2003.

MOENS, M.; PERRY, R. N.; STARR, J. L. Meloidogyne species: a diverse group of novel and important plant parasites. In: PERRY, R. N. et al. (Eds.). Root-knot nematodes. Wallingford: CABI Publishing, 2009. p. 1-17.

NICO, A. I.; JIMÉNEZ-DIAZ, R. M.; CASTILLO, P. Control of root-knot nematodes by composted agroindustrial wastes in potting mixtures. Crop Protection, Amsterdam, v. 23, n. 7, p. 581-587, 2004.

OYEDEMI, S. O. et al. The proposed mechanism of bactericidal action of eugenol, $\alpha$-terpineol and $\gamma$-terpinene against Listeria monocytogenes, Streptococcus pyogenes, Proteus vulgaris and Escherichia coli. African Journal of Biotechnology, Abraka, v. 8, n. 7, p. 1280-1286, 2009. 
PARK, I. K. et al. Nematicidal activity of plant essential oils and components from ajowan (Trachyspermum ammi), allspice (Pimenta dioica) and litsea (Litsea cubeba) essential oils against pine wood nematode (Bursaphelenchus Xylophilus). Journal of Nematology, Hanover, v. 39, n. 3, p. 275-279, 2007.

SALGADO, S. M. L.; SILVA, L. H. C. P. Potencial da indução de resistência no controle de fitonematoides. In: CAVALCANTI, L. S. et al. (Eds.). Indução de resistência em plantas a patógenos e insetos. Piracicaba: Fealq, 2005. p. 155-168.

SCHWAN-ESTRADA, K. R. F.; STANGARLIN, J. R.; CRUZ, M. E. S. Uso de plantas medicinais no controle de doenças de plantas. Fitopatologia Brasileira, Brasília, DF, v. 28, n. 1, p. 54-56, 2003.
SILVA, F. A. S. Software Assistat: assistência estatística. Versão 7.6 beta. Campina Grande: UAEG-CTRN-UFCG, 2011.

TSAO, R.; YU, Q. Nematicidal activity of monoterpenoid compounds against economically important nematodes in agriculture. Journal of Essential Oil Research, Messina, v. 12, n. 3, p. 350-354, 2000.

ZAMBOLIM, L. Tipos de fungicidas empregados no controle de doenças de plantas. In: ZAMBOLIM, L. et al. (Eds.). Produtos fitossanitários (fungicidas, inseticidas, acaricidas e herbicidas). Viçosa: UFV/DFP, 2008. p. 263348. 